Article

\title{
Quercetin, a Promising Clinical Candidate for The Prevention of Contrast-Induced Nephropathy
}

\author{
Laura Vicente-Vicente 1,2,3 , David González-Calle ${ }^{4}$, Alfredo Ginés Casanova 1,2,3, \\ María Teresa Hernández-Sánchez ${ }^{1,2,3}$, Marta Prieto 1,2,3, Juan Carlos Rama-Merchán ${ }^{4}$, \\ Javier Martín-Moreiras ${ }^{2,4}$, Francisco Martín-Herrero ${ }^{2,4}$, Pedro Luis Sánchez 2,4 (D), \\ Francisco J. López-Hernández ${ }^{1,2,3}$, Ignacio Cruz-González ${ }^{2,4}$ and Ana Isabel Morales $1,2,3, *$ (D) \\ 1 Toxicology Unit, University of Salamanca, 37007 Salamanca, Spain; lauravicente@usal.es (L.V.-V.); \\ alfredogcp@usal.es (A.G.C.); hsteresa@usal.es (M.T.H.-S.); martapv@usal.es (M.P.); \\ flopezher@usal.es (F.J.L.-H.) \\ 2 Institute of Biomedical Research of Salamanca (IBSAL), 37007 Salamanca, Spain; \\ jmmoreiras@gmail.com (J.M.-M.); fmartinherrero@usal.es (F.M.-H.); pedrolsanchez@secardiologia.es (P.L.S.); \\ cruzgonzalez.ignacio@gmail (I.C.-G.) \\ 3 Group of Translational Research on Renal and Cardiovascular Diseases (TRECARD), 37007 Salamanca, Spain \\ 4 Department of Cardiology, Hospital Universitario de Salamanca, 37007 Salamanca, Spain; \\ davidcalle1990@hotmail.com (D.G.-C.); ramamerchan@hotmail.com (J.C.R.-M.) \\ * Correspondence: amorales@usal.es; Fax: +34-923-294-669
}

Received: 28 August 2019; Accepted: 4 October 2019; Published: 8 October 2019

\begin{abstract}
Iodinated contrast media (CM) are the leading cause of acute renal failure of toxic origin. Between $21 \%$ and $50 \%$ of patients that receive them develop contrast-induced nephropathy (CIN). All prophylactic measures used so far have failed to provide effective prevention. Since oxidative stress is involved in the damage, a possible preventive strategy could be the administration of antioxidant substances, such as quercetin. This compound has shown renoprotective effects in experimental studies. The aim of this study was to evaluate whether quercetin may be helpful in preventing CIN in patients undergoing coronary catheterization. A clinical phase II study was conducted. Patients were distributed in two groups, namely, $\mathrm{CM}$ (patients who only received contrast media) and $\mathrm{CM}+\mathrm{Q}$ (patients who were pretreated with quercetin orally for 3-5 days). Results showed less incidence of $\mathrm{CIN}$ in the $\mathrm{CM}+\mathrm{Q}$ group, possibly due to glomerular protection, evidenced by a lower increase in serum creatinine and albuminuria; and a lower decrease in the glomerular filtration rate (GFR). Furthermore, in this group, the relative risk of developing CIN observed in patients that received a high dose of contrast media was inferior. In conclusion, this is the first study that demonstrates that quercetin is a promising safe candidate in preventing CIN.
\end{abstract}

Keywords: quercetin; glomerular injury; contrast media; contrast-induced nephropathy; prevention; flavonoids; albuminuria

\section{Introduction}

Contrast-induced nephropathy (CIN) is the major complication related to cardiac diagnostic or interventional procedures that require the use of contrast media (CM). The European Society of Urogenital Radiology defines CIN as an increase in serum creatinine of $\geq 0.5 \mathrm{mg} / \mathrm{dL}$ or $\geq 25 \%$ from the baseline value within three days after $\mathrm{CM}$ administration, in the absence of other causes for impaired renal function [1]. CIN is the third most common cause of acute renal failure acquired in the hospital [2] and it is associated with significant morbidity and mortality in the short and long term $[3,4]$. This condition has also been linked to longer in-hospital stay and higher incidence of chronic renal failure [5]. 
CIN pathogenesis is not fully understood and seems to be due to different factors. The intravenous injection of CM induces an initial increase in the glomerular filtration rate (GFR) due to a transient afferent arteriolar vasodilation. Subsequently, a prolonged vasoconstriction occurs, leading to a sustained decrease in the GFR over time, whereby a renal ischemia occurs. This state of hypoxia results in the formation of reactive oxygen species that can produce tubular damage and further intensify the reduction of the GFR. Because CM are excreted through the kidney, renal tubular cells are directly exposed to the toxicant. That is why, in preclinical studies, massive intratubular necrosis and tubular obstruction have been observed [6].

Only two procedures have shown a relative efficacy in preventing CIN in the clinical setting-the reduction of $\mathrm{CM}$ volume and isotonic hydration prior to catheterization. Other approaches such as the administration of sodium bicarbonate [7] or diuretics [8] have also been tested, without effective results. Thus, these measures are not enough, and a large number of patients continue developing CIN. For this reason, new strategies to reduce CIN incidence and severity are being pursued. Specifically, animal studies are testing nephroprotective substances designed to either facilitate CM removal in order to prevent their accumulation in renal tubules, to inhibit tubular CM uptake, or to form less toxic complexes, or to modulate signaling pathways involved in their toxic effect. However, these compounds are still in preclinical stages and the effect on humans is unknown [9-11]. A possible solution to this problem could be the administration of antioxidants since, as previously mentioned, the formation of free radicals seems to play an important role in the pathogenesis of renal damage caused by CM. An antioxidant candidate as nephroprotector could be quercetin. This compound is an abundant vegetal flavonoid in the Mediterranean diet to which several properties have been attributed, such as antioxidant, anti-inflammatory, analgesic, antihistamine, cholesterol reducer, antiviral, and renal hemodynamic modulator (reviewed in Vicente-Vicente et al., 2013 [12]). In addition, preclinical studies have been published supporting the conclusion that quercetin exerts protection against renal damage induced by cadmium chronically administered, due to its properties as antioxidant, anti-inflammatory, vasodilator, and metallothionein inductor $[13,14]$. It has also proven to be effective in preventing nephrotoxicity caused by the antineoplastic cisplatin [15]. Its renoprotective effect has also been evaluated in vitro [16]. In tubular epithelial cells, quercetin was linked to decreased lipid peroxidation and free radical removal. It was also tested as a nephroprotector in an ischemia/reperfusion model, in which the increased nitric oxide concentration was reduced by quercetin pretreatment [17].

Regarding quercetin safety in humans, a large number of clinical studies have been conducted both in phase I and phase II, testing several doses of quercetin [18-29]. Only in one clinical trial have some adverse effects associated with this product been reported. The intravenous administration of a high dose $(51.3 \mathrm{mg} / \mathrm{kg})$ caused dyspnea, emesis, and nephrotoxicity in one patient [25]. None of the studies in which quercetin was administered orally has reported adverse effects, indicating that quercetin is safe for human administration [17-19,21,23,24,26-28], even at doses of $1500 \mathrm{mg} /$ day for 84 days [20].

With the arguments above, quercetin can be considered as a promising candidate as nephroprotectant against CIN. Therefore, the objectives of this work were to study the effectiveness of the flavonoid quercetin as a novel strategy to reduce the incidence of CIN in cardiac patients and to evaluate its possible renal protective effect through the determination of tubular and glomerular damage biomarkers.

\section{Results}

\subsection{Patient Characteristics}

During the recruitment period, 192 patients agreed to participate in the study, of which 134 were included in the CM group and 58 in the $C M+Q$ group. Sample collection was done in two phases; first, control (CM group) samples were collected and, second, the samples of patients receiving quercetin $(C M+Q)$ were collected. Although the collection period was similar in both groups, fewer patients 
agreed to take quercetin. For this reason, the number of patients was different between the groups. Despite this, anthropometric characteristics and risk factors were homogeneous in both groups (Table 1). Regarding pharmacological treatment in each group, no differences were observed between the two groups. The most commonly consumed drugs were those related to the pathologies mentioned as risk factor (i.e., antihypertensives, cholesterol-lowering drugs, and antidiabetics. Since patients had cardiac pathologies, it was also observed that almost half of the patients in each group consumed antiplatelet agents (acetyl salicylic acid (ASA) and others). It is remarkable that a high percentage of patients in the $\mathrm{CM}+\mathrm{Q}$ group (17.0\%) consumed non-steroidal anti-inflammatory drugs (NSAIDs), whereas in the CM group only $7.3 \%$ consumed them (Table 2 ).

Table 1. Anthropometric characteristics, risk factors associated with contrast media-induced nephropathy of patients in each study group, and information related to the contrast agent administered (for each type, percentage of patients, and volume). CM, patients who were administered iodinated contrast media; $\mathrm{CM}+\mathrm{Q}$, patients pretreated with quercetin. ${ }^{*} p<0.05$ vs. CM group. BMI, body mass index; SEM, standard error of the mean.

\begin{tabular}{|c|c|c|}
\hline & \multicolumn{2}{|c|}{ Group } \\
\hline & CM Group & $\mathrm{CM}+\mathrm{Q}$ Group \\
\hline \multicolumn{3}{|c|}{ Anthropometric Characteristics } \\
\hline Men (\%) & 77.6 & 67.3 \\
\hline Women $(\%)$ & 22.4 & 32.7 \\
\hline $\begin{array}{l}\text { Age (years; mean } \pm \text { SEM) } \\
\text { (minimum-maximum) }\end{array}$ & $\begin{array}{c}71.4 \pm 1.1 \\
(39-91)\end{array}$ & $\begin{array}{c}68.5 \pm 1.4 \\
(46-88)\end{array}$ \\
\hline BMI (mean \pm SEM) & $27.6 \pm 0.4$ & $31.8 \pm 3.7$ \\
\hline \multicolumn{3}{|c|}{ Risk Factors } \\
\hline Diabetes mellitus (\%) & 27.6 & 31.0 \\
\hline Dyslipidemia (\%) & 44.0 & 51.7 \\
\hline Arterial hypertension (\%) & 56.7 & 53.4 \\
\hline Smoking (\%) & 20.1 & $36.2 *$ \\
\hline \multicolumn{3}{|c|}{ Type of Contrast Agent } \\
\hline \multicolumn{3}{|l|}{$\mathrm{CM}$ administered $(\%) /$} \\
\hline \multicolumn{3}{|l|}{ Volume $\mathrm{mL}($ mean $\pm \mathrm{SEM})$} \\
\hline Iodixanol & $\begin{array}{c}70.1 / \\
295.0 \pm 15.0\end{array}$ & $\begin{array}{c}63.8 / \\
274.1 \pm 18.7\end{array}$ \\
\hline Iohexol & $\begin{array}{c}22.4 / \\
260.0 \pm 21.7\end{array}$ & $\begin{array}{c}25.9 / \\
308.4 \pm 28.0\end{array}$ \\
\hline Iodine (not specified) & $\begin{array}{c}0.8 / \\
100.0 \pm 0.0\end{array}$ & $\begin{array}{c}3.5 / \\
600.0 \pm 0.0\end{array}$ \\
\hline Without data & 6.7 & 5.2 \\
\hline
\end{tabular}


Table 2. Drug consumption before contrast media administration. ACE, angiotensin-converting enzyme; ARBs, angiotensin II receptor blockers; ASA, acetyl salicylic acid; NSAIDs, non-steroidal anti-inflammatory drugs. Data are expressed as percentage of patients. No statistical differences were observed between the groups.

\begin{tabular}{|c|c|c|}
\hline Pharmacological Group & CM Group & $\mathrm{CM}+\mathrm{Q}$ Group \\
\hline \multicolumn{3}{|c|}{ Hypertension Treatment } \\
\hline ACE inhibitors & 26.9 & 27.6 \\
\hline Aldosterone receptor antagonists & 0.8 & 2.12 \\
\hline Alpha blockers & 4.1 & 10.6 \\
\hline ARBs & 15.4 & 17.0 \\
\hline Beta blockers & 26.9 & 19.1 \\
\hline Calcium channels antagonists & 17.9 & 4.2 \\
\hline Diuretics & 28.5 & 23.4 \\
\hline \multicolumn{3}{|c|}{ Dyslipidemia Treatment } \\
\hline Statins & 38.2 & 31.9 \\
\hline \multicolumn{3}{|c|}{ Diabetes Mellitus Treatment } \\
\hline Antidiabetics & 21.1 & 21.3 \\
\hline \multicolumn{3}{|c|}{ Thrombus Prevention Treatment } \\
\hline ASA & 35.8 & 31.9 \\
\hline Antiplatelet agents & 25.2 & 12.8 \\
\hline \multicolumn{3}{|c|}{ Others } \\
\hline NSAIDs & 7.3 & 17.0 \\
\hline
\end{tabular}

\subsection{Basal Renal Function}

The baseline serum creatinine, urea, and the GFR were similar in both groups as shown in Table 3 $(p>0.05)$.

Table 3. Basal renal function in both groups. Crs, serum creatinine; eGFR, estimated glomerular filtration rate; CKD-EPI, Chronic Kidney Disease Epidemiology Collaboration. Data are expressed as mean \pm standard error of the mean. No significant differences were found $(p>0.05)$.

\begin{tabular}{ccc}
\hline & CM Group & CM+Q Group \\
\hline Crs $(\mathrm{mg} / \mathrm{dL})$ & $1.08 \pm 0.04$ & $0.96 \pm 0.04$ \\
\hline Serum urea $(\mathrm{mg} / \mathrm{dL})$ & $52.20 \pm 2.54$ & $48.91 \pm 3.00$ \\
\hline eGFR CKD-EPI $\left(\mathrm{mL} / \mathrm{min} / 1.73 \mathrm{~m}^{2}\right)$ & $82.61 \pm 1.09$ & $85.93 \pm 1.44$ \\
\hline
\end{tabular}

\subsection{Quercetin Efficacy as Nephroprotector}

First, CIN incidence was calculated in each group (Figure 1), by calculating the percentage of patients whose creatinine increase was greater than $0.5 \mathrm{mg} / \mathrm{dL}$ or $25 \%$ from the baseline value. The CIN incidence was lower in the $\mathrm{CM}+\mathrm{Q}$ group $(13.8 \%$ in $\mathrm{CM}+\mathrm{Q}$ versus $17.2 \%$ in $\mathrm{CM})$. 


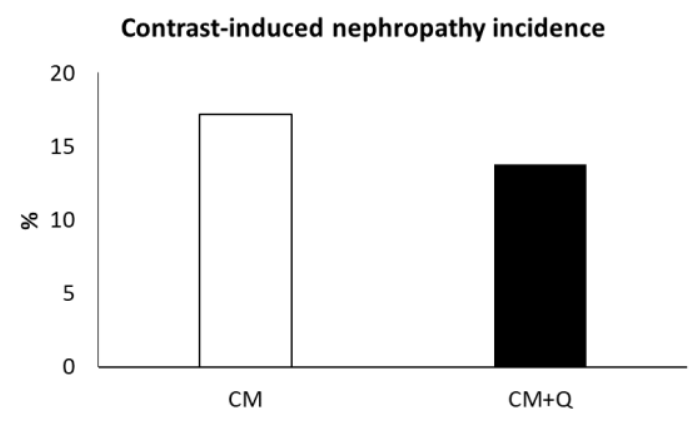

Figure 1. Contrast-induced nephropathy (CIN) incidence, calculated as \% of patients whose increase in serum creatinine during the three days post contrast media was higher than $0.5 \mathrm{mg} / \mathrm{mL}$ or $25 \%$ with respect to its basal value. No significant differences were found $(p>0.05)$.

Subsequently and with the aim of assessing the ability of quercetin to prevent kidney injury caused by exposure to $C M$, the increases in serum creatinine (Figure 2a), proteinuria (Figure $2 b$ ), urinary $\mathrm{N}$-acetyl- $\beta$-D-glucosaminidase (NAG; Figure $3 \mathrm{a}$ ), urinary neutrophil gelatinase-associated lipocalin (NGAL; Figure 3b), urinary kidney injury molecule 1 (KIM-1; Figure 3c), and albuminuria (Figure 3d) were calculated, as well as the decrease in the glomerular filtration rate (GFR; Figure 2c) using the Chronic Kidney Disease Epidemiology Collaboration (CKD-EPI) formula. A lower increase in serum creatinine concentration and albuminuria was observed in patients treated with quercetin when compared with untreated patients (serum creatinine increase: $7.62 \% \pm 3.49 \%$ in $\mathrm{CM}+\mathrm{Q}$ versus $11.91 \% \pm 3.43 \%$ in $\mathrm{CM}$, $p$-value $=0.97$; albuminuria increase: $38.3 \% \pm 33.5 \%$ in $\mathrm{CM}+\mathrm{Q}$ versus $282.9 \% \pm 84.0 \%$ in $\mathrm{CM}, p$-value $=0.02)$. No differences in proteinuria, NAG, NGAL, and KIM-1 were observed between groups ( $p>$ 0.05). However, the GFR showed a greater decrease in patients who did not receive quercetin, which is consistent with the results of plasma creatinine.
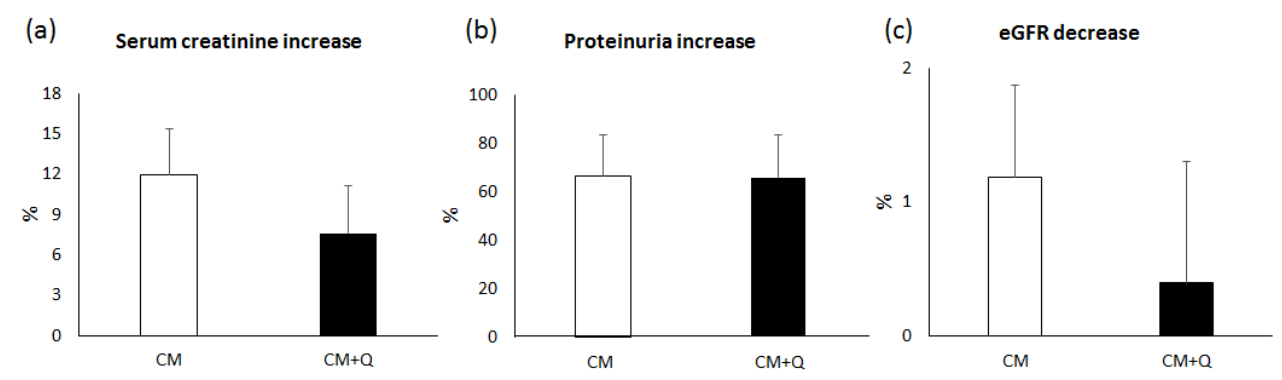

Figure 2. Renal function assessment in each study group. CM, patients who were administered iodinated contrast media; $\mathrm{CM}+\mathrm{Q}$, patients receiving iodinated contrast media and quercetin. (a) Increase in serum creatinine; (b) Increase in proteinuria; (c) Decrease in eGFR calculated using the Chronic Kidney Disease Epidemiology Collaboration (CKD-EPI) formula. Increases or decreases were calculated as $\%$ using basal biomarker data and the highest value observed during the $72 \mathrm{~h}$ after contrast media administration. Data are expressed as mean \pm standard error of the mean. No significant differences were found $(p>0.05)$. eGFR, estimated glomerular filtration rate 

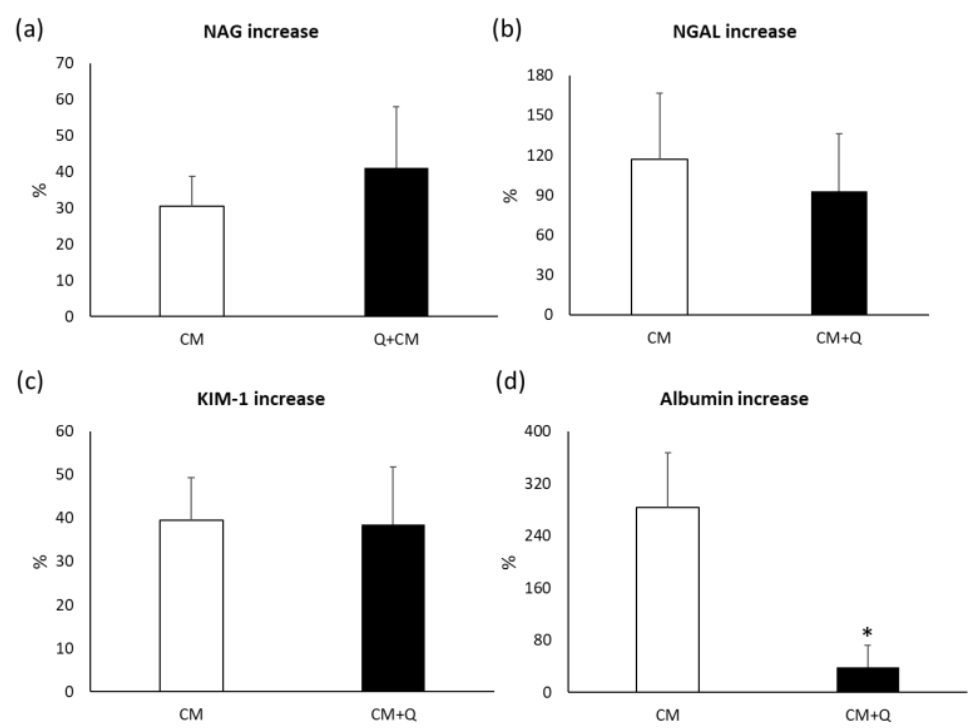

Figure 3. Quantification of early kidney damage biomarkers in each study group. CM, patients who were administered iodinated contrast media; $C M+Q$, patients receiving iodinated contrast media and quercetin. (a) Increase in urinary NAG; (b) Increase in urinary NGAL; (c) Increase in urinary KIM-1; (d) Increase in urinary albumin. Increase was calculated as \% using basal biomarker data and the highest value observed during the $72 \mathrm{~h}$ after contrast media administration. Data are expressed as mean \pm standard error of the mean. ${ }^{*} p<0.05$ vs. CM group. KIM-1, kidney injury molecule 1 ; NAG, $\mathrm{N}$-acetyl- $\beta$-D-glucosaminidase; NGAL, neutrophil gelatinase-associated lipocalin.

\subsection{Relative Risk of CIN from Each Risk Factor}

In each study group, the relative risk (RR) of developing CIN was calculated for each parameter considered as a risk factor (Table 4). RR indicates the probability of an event occurring (in this study, the event is CIN) by comparing the number of patients that present the risk factor (arterial hypertension, dyslipidemia, etc.) with those who do not present it. A higher value indicates a higher probability of developing CIN because of the risk factor. Values were very similar in both groups although slightly lower in the $\mathrm{CM}+\mathrm{Q}$ group, except for the RR calculated with the volume of $\mathrm{CM}$. Administration of more than $350 \mathrm{~mL}$ was associated with a greater number of CIN cases (more than twice) in patients in the $\mathrm{CM}$ group than in patients that received quercetin.

Table 4. Calculation of the relative risk to develop contrast-induced nephropathy for each risk factor. $R R$, relative risk; $C M$, patients who were administered iodinated contrast media; $C M+Q$, patients pretreated with quercetin before CM administration. * Statistically significant $(p<0.05)$.

\begin{tabular}{|c|c|c|c|c|c|c|}
\hline \multirow{3}{*}{ Risk Factor } & \multicolumn{3}{|c|}{$\mathrm{CM}$} & \multicolumn{3}{|c|}{$\mathrm{CM}+\mathrm{Q}$} \\
\hline & \multirow{2}{*}{ RR } & \multicolumn{2}{|c|}{ 95\% Confidence Interval } & \multirow{2}{*}{$\mathbf{R R}$} & \multicolumn{2}{|c|}{ 95\% Confidence Interval } \\
\hline & & Lower Limit & Upper Limit & & Lower Limit & Upper Limit \\
\hline Arterial hypertension & 1.19 & 0.83 & 1.68 & 0.87 & 0.41 & 3.85 \\
\hline Diabetes mellitus & 1.15 & 0.57 & 2.29 & 0.74 & 0.21 & 2.63 \\
\hline Dyslipidemia & 0.73 & 0.41 & 1.32 & 1.56 & 0.85 & 2.82 \\
\hline Smoking & 1.17 & 0.50 & 2.71 & 1.06 & 0.40 & 2.78 \\
\hline $\mathrm{CM}$ volume $>350 \mathrm{~mL}$ & $1.83 *$ & 1.07 & 3.14 & 0.80 & 0.22 & 2.86 \\
\hline
\end{tabular}

\subsection{Quercetin Safety}

The flavonoid administration at a dose of $500 \mathrm{mg}$ every $8 \mathrm{~h}$ for 3-5 days produced no adverse effects, as shown by the results of the surveys conducted in quercetin-treated group, in which only $3 \%$ of patients reported moderate gastrointestinal pain. 


\section{Discussion}

CM administration has increased in recent years [30]. This fact, along with the elevated prevalence of CIN risk factors (chronic kidney disease and diabetes mellitus), represents an immediate urgency in the search for strategies that can prevent the development of kidney damage following CM administration.

It is known that oxidative stress plays an important role in the pathogenesis of CM-induced renal damage [6]. In this context, this study proposed the prophylactic treatment with the antioxidant quercetin as a possible solution.

Our data demonstrate that the administration of quercetin is able to attenuate the increase in albumin excretion. If albuminuria truly reflects a malfunction in the glomerular filtration barrier [31], then this study lends credence to the hypothesis that quercetin may be protective through its biological effect ameliorating oxidative damage at the level of the glomerular apparatus [32]. This finding has even been confirmed in studies with other antioxidants such as N-acetyl-cysteine against CIN [33]. It should also be noted that the protective capacity of quercetin is evident even though the percentage of smokers was higher in the CM+Q group. Since tobacco contributes to oxidative stress generation, it is possible that if the prevalence of this risk factor had been similar in both groups, the observed nephroprotective effect would have been greater.

Oxidative stress protection could justify the lower increase in serum creatinine and the lower incidence of CIN in patients who were treated with quercetin. Regarding creatinine, the lack of statistical significance in this parameter could be because creatinine measurement is too insensitive to detect minor insults to the glomeruli. On the other hand, albuminuria has a greater capacity to detect kidney damage in initial stages [34], increasing its urine excretion earlier and more specifically than serum creatinine, which would explain the higher difference. Regarding the incidence of CIN, it was $17.2 \%$ in the patients who only received the CM, in agreement with data reported for patients with heart disease and similar risk factors shown by our study group $[2,35,36]$. The incidence was lower $(13.8 \%)$ in the CM+Q group. The calculation of the GFR using the CKD-EPI formula showed a greater decrease in patients who did not receive quercetin, which is consistent with the results described above.

In order to evaluate the possible protective effect of quercetin on contrast-induced tubular damage, the excretion of some specific markers such as NAG, NGAL, and KIM-1 was measured. NAG is an enzyme which is released to the urine after an injury in the tubular cells. Clinical studies have shown that its urinary excretion increase in the context of a variety of drugs, including contrast media, occurs earlier than the increase in serum creatinine [37-39]. NGAL is a protein whose expression in the kidney is induced as a consequence of hypoxic damage, especially in proximal tubular cells [40]. Previous studies have shown a significant increase of this protein in urine $2 \mathrm{~h}$ after CM administration [41]. On the other hand, KIM-1 is a protein that confers to cells the ability to phagocytose dead cells after an ischemic injury of the kidney [42]. Its utility to diagnose CIN has not been widely studied. In accordance with these studies, our results show that CM exert tubular toxicity, although quercetin appears to have no capacity to protect at this level, since the increase is similar in both groups studied (Figure 3).

The definition of CIN determines that renal damage occurs as a consequence of CM administration. It is necessary to take into account that the presence of other risk factors (hypertension, diabetes, consumption of NSAIDs, etc.) can increase the risk of suffering CIN; therefore, the RR for CIN because of each risk factor was calculated. The value of RR to suffer CIN was similar in all evaluated risk factors in both groups, indicating that quercetin does not influence any of these parameters. However, the RR calculated in patients whose CM volume is greater than $350 \mathrm{~mL}$ showed that the risk of CIN due to the administration of high amounts of CM is lower in patients receiving quercetin. These data are very important and suggest that quercetin may reduce the risk of CIN derived from CM administration, allowing the management of larger volumes of CM without increasing the risk of CIN, which may be necessary in diagnostic or surgical interventions. 
Other data collected during the study were the days of hospital stay and mortality. In both groups, the mean number of days admitted to the hospital was 6 . In terms of mortality, $14.7 \%$ of patients in the $\mathrm{CM}$ group died some days after $\mathrm{CM}$ administration, whereas in $\mathrm{CM}+\mathrm{Q}$ this number decreased to $7.8 \%$. Although it is a very important difference, it cannot be completely attributed to flavonoid administration since it may be due to other factors related to the pathology according to which the patient was operated. Even so, the reduction of CIN by quercetin could be involved in this lower number of deaths, but confirming the hypothesis would require a greater number of patients.

On the other hand, no toxic effects were associated with the use of quercetin, except for abdominal pain which was reported by $3 \%$ of patients treated with this substance. With these data, it could be argued that the oral administration of quercetin at a dose of $1500 \mathrm{mg}$ /day is safe in cardiac patients with associated risk factors. This result is in agreement with the information contained in the literature, where the oral administration of quercetin was safe, both in the healthy population $[18,19,24]$ and in patients with different diseases such as gastrointestinal diseases [28] or cardiovascular diseases, hypertension, metabolic syndrome, and high cholesterolemia [20,21,43,44].

Considering all the above, these results suggest a potential effectiveness of quercetin as nephroprotector against CM-induced renal damage with absence of adverse effects. However, some limitations of this study make it difficult to repeat in humans the results obtained in preclinical studies [13-15]. The main limitation was the poor oral bioavailability of flavonoids [45]. In laboratory animals, the i.p. mode was used, which allows the administration of the highest doses of quercetin in comparison to the oral mode used in patients. Moreover, the present data cannot be compared with previous works since this is the first study designed to assess the use of quercetin as a human nephroprotective substance. It is possible that if the bioavailability of quercetin increases, a greater protection will be provided against renal damage produced by $\mathrm{CM}$. The study of new formulations of quercetin with greater absorption could be a good solution. Other limitations that may have affected the study are, first, that the $\mathrm{CM}$ group did not receive the oral formulation without quercetin, so the placebo effect was not contemplated. However, in this work, it was considered that this did not interfere since the efficacy was evaluated through objective biochemical parameters. Second, it would have been of special interest to know the blood levels of quercetin in each patient in order to associate the flavonoid concentration with the observed protective effect. However, the necessary techniques to carry this out were not available.

This study suggests that quercetin may attenuate glomerular injury in patients who received CM and were pretreated with this flavonoid. The administration of quercetin appears to be a promising candidate for the prevention of CIN. Thus, this study is a first possible step for including quercetin as a prophylactic measure in CIN management protocols.

\section{Materials and Methods}

\subsection{Study Population}

The experimental protocol was approved by the "Ethics Committee of research with medicines of Salamanca" of the "Complejo Asistencial Universitario de Salamanca" (Salamanca, Spain), reference number PI 201103001 (2 May 2011). All the following procedures were performed in accordance with the ethical standards of the responsible committee on human experimentation (institutional and national) and with the Helsinki Declaration of 1975, as revised in 2000. Informed consent was obtained from all patients included in the study.

In this randomized prospective study, patients suffering from acute coronary syndrome without ST elevation who would receive iodinated CM for diagnostic procedures were enrolled. It was estimated that, in order to obtain results that were statistically relevant, at least 50 patients treated with quercetin and their respective controls (patients with the same conditions but without quercetin) would be required. Patients who agreed to participate met the inclusion criteria (be over 18 years old and sign an informed consent). Two experimental groups were made, namely, Group CM (patients who only 
received $C M$ ) and Group $C M+Q$ (patients who were pretreated with quercetin orally (500 mg/8 h)). The planned posology was to start quercetin administration two days before $\mathrm{CM}$ administration and continue the following two days, but due to the urgency of some catheterizations the total posology of quercetin ranged between 3 and 5 days.

\subsection{Samples and Clinical Data Collection}

Blood and urine samples were collected immediately before CM administration (basal) and 24, 48, and $72 \mathrm{~h}$ after $\mathrm{CM}$. These samples were centrifuged and stored at $-80^{\circ} \mathrm{C}$.

For each participant, the following data were collected: age, gender, body mass index, risk factors associated with CIN (diabetes mellitus, arterial hypertension, and smoking), CM data (CM administered and volume), and pharmacological treatment received before/during $\mathrm{CM}$ administration.

\subsection{Renal Function Evaluation}

Renal function was determined at time points $0,24,48$, and $72 \mathrm{~h}$ by quantifying serum creatinine and urea concentrations, proteinuria, GFR, and urinary NAG, NGAL, KIM-1, and albumin excretion. The increase (in percentage) of each parameter studied was calculated using the following formula:

$$
\text { Biomarker increase }(\%)=(\text { Maximum value }- \text { Basal value }) /(\text { Basal value }) \times 100
$$

where Maximum value means the highest value reached by the parameter during the first $72 \mathrm{~h}$ after $\mathrm{CM}$, whereas Basal value means the value of the biomarker before $\mathrm{CM}$ administration.

Serum creatinine was evaluated using an automatic analyzer (Roche/Hitachi 917; Mannheim, Germany). It was considered that the patient suffered CIN if creatinine increased $>25 \%$ of its basal value in the first $72 \mathrm{~h}$ after $\mathrm{CM}$ administration [1]. The same analyzer was used to measure serum urea. The CKD-EPI formula [46] was used to estimate the GFR.

Urinary excretion of proteins was determined by the Bradford colorimetric method [47]. Urinary NAG activity was measured using a commercial kit ("N-Acetyl- $\beta$-D-glucosaminidase NAG assay kit DZ062A-K", Diazyme, Poway, CA, USA), following the manufacturer's instructions. Urinary levels of NGAL, KIM-1, and albumin were measured by commercial ELISAs ("Human NGAL ELISA Kit 036CE”, BioPorto Diagnostics, Hellerup, Denmark; "Human KIM-1 ELISA Kit \#ADI-900-226”, Enzo Life Sciences, Farmingdale, NY, USA; and "Human Albumin ELISA Kit E88-129", Bethyl Laboratories, Montgomery, TX, USA, respectively), according to the manufacturer's instructions. All urinary biomarkers quantified were corrected by the concentration of creatinine in urine.

\subsection{Statistical Analysis}

Results are presented as mean \pm standard error of the mean (SEM). Data were statistically analyzed using the IBM SPSS Statistics 20 software (Chicago, IL, USA). Because the results were not normally distributed (according to the Kolmogorov-Smirnov test), they were analyzed using the nonparametric Mann-Whitney test in order to check statistically significant differences between groups. To compare qualitative data, a Pearson's chi-squared test was applied to evaluate the homogeneity between groups. It was considered a significant difference when $p<0.05$.

\subsection{Relative Risk (RR) of CIN Calculation}

For each risk factor (diabetes mellitus, dyslipidemia, arterial hypertension, and smoking), the RR of developing CIN was calculated in both groups, with the aim of studying if quercetin administration reduced or not the probability to develop CIN due to the risk factor. In addition, the volume of CM administered was considered as another risk factor in several studies [48-50]. For this reason, in each group, patients were separated into two groups, one for those who were given less than $350 \mathrm{~mL}$ and another for those who were given $350 \mathrm{~mL}$ or more. 
RR (with its 95\% confidence interval) was calculated for each risk factor using the following formula:

$$
R R=(a /(a+b)) /(c /(c+d))
$$

where $a$ is the number of patients that develop CIN and have the risk factor; $b$ is the number of patients without CIN but with the risk factor; $c$ is the number of patients that develop CIN but do not have the risk factor; and $d$ is the number of patients without CIN nor risk factor.

The statistical significance was inferred at a two-sided $p$-value $<0.05$.

\subsection{Quercetin Safety}

To ensure the safety of quercetin, the nursing service was responsible for monitoring the health of patients during and after treatment with quercetin. In addition, a questionnaire about adverse effects associated with the consumption of this flavonoid was distributed. Specifically, patients were questioned about headaches, palpitations, diarrhea, fatigue, edema, nausea or vomiting, abdominal pain, dizziness, and fever.

Author Contributions: Study conception and design, A.I.M. and I.C.-G.; Patient recruitment and sample collection, J.C.R.-M., J.M.-M., F.M.-H., and P.L.S.; Sample processing and analysis, L.V.-V., A.G.C., M.T.H.-S., and M.P.; Database development and statistical analysis, L.V.-V., A.G.C., and D.G.-C.; Writing-original draft, L.V.-V. and F.J.L.-H.; Writing-review and editing, A.I.M. and I.C.-G.; Supervision, A.I.M.

Funding: Research from the authors' laboratory supporting part of the information incorporated into this article was funded by grants from Junta de Castilla y León (Consejería de Sanidad, BIO/SA66/15, and Consejería de Educación, SA359U14); from Fundación Mutua Madrileña (2012 call); from Instituto de Salud Carlos III (PI17/01979, DT15S/00166, and PI15/01055, PI14/01776); and co-funded by FEDER and Retic (RD016/0009/0025) REDINREN-FEDER funds.

Conflicts of Interest: The authors declare no conflict of interest.

\section{Abbreviations}

$\begin{array}{ll}\text { ACE } & \text { Angiotensin converting enzyme } \\ \text { ARBs } & \text { Angiotensin II receptor blockers } \\ \text { ASA } & \text { Acetyl salicylic acid } \\ \text { BMI } & \text { Body mass index } \\ \text { CIN } & \text { Contrast-induced nephropathy } \\ \text { CKD-EPI } & \text { Chronic Kidney Disease Epidemiology Collaboration } \\ \text { CM } & \text { Contrast media } \\ \text { Crs } & \text { Serum creatinine } \\ \text { eGFR } & \text { Estimated glomerular filtration rate } \\ \text { GFR } & \text { Glomerular filtration rate } \\ \text { KIM-1 } & \text { Kidney injury molecule 1 } \\ \text { NAG } & \text { N-acetyl- } \beta \text {-D-glucosaminidase } \\ \text { NGAL } & \text { Neutrophil gelatinase-associated lipocalin } \\ \text { NSAIDs } & \text { Nonsteroidal anti-inflammatory drugs } \\ \text { Q } & \text { Quercetin } \\ \text { RR } & \text { Relative risk } \\ \text { SEM } & \text { Standard error of the mean }\end{array}$

\section{References}

1. Morcos, S.K.; Thomsen, H.S. European Society of Urogenital Radiology guidelines on administering contrast media. Abdom Imaging 2003, 28, 0187-0190. [CrossRef] [PubMed]

2. Andreucci, M.; Faga, T.; Pisani, A.; Sabbatini, M.; Michael, A. Acute kidney injury by radiographic contrast media: Pathogenesis and prevention. Biomed. Res. Int. 2014, 2014, 362725. [CrossRef] [PubMed] 
3. Marenzi, G.; Lauri, G.; Assanelli, E.; Campodonico, J.; Metrio, M.D.; Marana, I.; Grazi, M.; Veglia, F.; Bartorelli, A.L. Contrast-induced nephropathy in patients undergoing primary angioplasty for acute myocardial infarction. J. Am. Coll. Cardiol. 2004, 44, 1780-1785. [CrossRef] [PubMed]

4. Rihal, C.S.; Textor, S.C.; Grill, D.E.; Berger, P.B.; Ting, H.H.; Best, P.J.; Singh, M.; Bell, M.R.; Barsness, G.W.; Mathew, V.; et al. Incidence and Prognostic Importance of Acute Renal Failure After Percutaneous Coronary Intervention. Circulation 2002, 105, 2259-2264. [CrossRef] [PubMed]

5. Best, P.J.M.; Lennon, R.; Ting, H.H.; Bell, M.R.; Rihal, C.S.; Holmes, D.R.; Berger, P.B. The impact of renal insufficiency on clinical outcomes in patients undergoing percutaneous coronary interventions. J. Am. Coll. Cardiol. 2002, 39, 1113-1119. [CrossRef]

6. Caiazza, A.; Russo, L.; Sabbatini, M.; Russo, D. Hemodynamic and Tubular Changes Induced by Contrast Media. Biomed Res. Int. 2014, 2014, 1-7. [CrossRef] [PubMed]

7. Zoungas, S.; Ninomiya, T.; Huxley, R.; Cass, A.; Jardine, M.; Gallagher, M.; Patel, A.; Vasheghani-Farahani, A.; Sadigh, G.; Perkovic, V. Systematic Review: Sodium Bicarbonate Treatment Regimens for the Prevention of Contrast-Induced Nephropathy. Ann. Intern. Med. 2009, 151, 631. [CrossRef]

8. Solomon, R.; Werner, C.; Mann, D.; D’Elia, J.; Silva, P. Effects of Saline, Mannitol, and Furosemide on Acute Decreases in Renal Function Induced by Radiocontrast Agents. N. Engl. J. Med. 1994, 331, 1416-1420. [CrossRef]

9. Linkermann, A.; Heller, J.-O.; Prókai, A.; Weinberg, J.M.; De Zen, F.; Himmerkus, N.; Szabó, A.J.; Bräsen, J.H.; Kunzendorf, U.; Krautwald, S. The RIP1-kinase inhibitor necrostatin-1 prevents osmotic nephrosis and contrast-induced AKI in mice. J. Am. Soc. Nephrol. 2013, 24, 1545-1557. [CrossRef]

10. Quiros, Y.; Sánchez-González, P.D.; López-Hernández, F.J.; Morales, A.I.; López-Novoa, J.M. Cardiotrophin-1 Administration Prevents the Renal Toxicity of Iodinated Contrast Media in Rats. Toxicol. Sci. 2013, 132, 493-501. [CrossRef]

11. Ulusoy, S.; Ozkan, G.; Mungan, S.; Orem, A.; Yulug, E.; Alkanat, M.; Yucesan, F.B. GSPE is superior to NAC in the prevention of contrast-induced nephropathy: Might this superiority be related to caspase 1 and calpain 1? Life Sci. 2014, 103, 101-110. [CrossRef] [PubMed]

12. Vicente-Vicente, L.; Prieto, M.; Morales, A.I. Eficacia y seguridad de la quercetina como complemento alimenticio. Rev. De Toxicol. 2013, 30, 171-181.

13. Morales, A.I.; Vicente-Sánchez, C.; Jerkic, M.; Santiago, J.M.; Sánchez-González, P.D.; Pérez-Barriocanal, F.; López-Novoa, J.M. Effect of quercetin on metallothionein, nitric oxide synthases and cyclooxygenase-2 expression on experimental chronic cadmium nephrotoxicity in rats. Toxicol. Appl. Pharmacol. 2006, 210, 128-135. [CrossRef] [PubMed]

14. Morales, A.I.; Vicente-Sánchez, C.; Sandoval, J.M.S.; Egido, J.; Mayoral, P.; Arévalo, M.A.; Fernández-Tagarro, M.; López-Novoa,J.M.; Pérez-Barriocanal, F. Protective effect of quercetin on experimental chronic cadmium nephrotoxicity in rats is based on its antioxidant properties. Food Chem. Toxicol. 2006, 44, 2092-2100. [CrossRef] [PubMed]

15. Sanchez-Gonzalez, P.D.; Lopez-Hernandez, F.J.; Perez-Barriocanal, F.; Morales, A.I.; Lopez-Novoa, J.M. Quercetin reduces cisplatin nephrotoxicity in rats without compromising its anti-tumour activity. Nephrol. Dial. Transplant. 2011, 26, 3484-3495. [CrossRef] [PubMed]

16. Kuhlmann, M.K.; Burkhardt, G.; Horsch, E.; Wagner, M.; Köhler, H. Inhibition of oxidant-induced lipid peroxidation in cultured renal tubular epithelial cells (LLC-PK1) by quercetin. Free Radic. Res. 1998, 29, 451-460. [CrossRef] [PubMed]

17. Shoskes, D.A. Effect of bioflavonoids quercetin and curcumin on ischemic renal injury: A new class of renoprotective agents. Transplantation 1998, 66, 147-152. [CrossRef] [PubMed]

18. Askari, G.; Hajishafiee, M.; Ghiasvand, R.; Hariri, M.; Darvishi, L.; Ghassemi, S.; Iraj, B.; Hovsepian, V. Quercetin and Vitamin C Supplementation: Effects on Lipid Profile and Muscle Damage in Male Athletes. Int. J. Prev. Med. 2013, 4, S58-S62.

19. Broman-Fulks, J.J.; Canu, W.H.; Trout, K.L.; Nieman, D.C. The effects of quercetin supplementation on cognitive functioning in a community sample: A randomized, placebo-controlled trial. Ther. Adv. Psychopharmacol. 2012, 2, 131-138. [CrossRef]

20. Clifton, P.M. Effect of Grape Seed Extract and Quercetin on Cardiovascular and Endothelial Parameters in High-Risk Subjects. J. Biomed. Biotechnol. 2004, 2004, 272-278. [CrossRef] 
21. Conquer, J.A.; Maiani, G.; Azzini, E.; Raguzzini, A.; Holub, B.J. Supplementation with quercetin markedly increases plasma quercetin concentration without effect on selected risk factors for heart disease in healthy subjects. J. Nutr. 1998, 128, 593-597. [CrossRef] [PubMed]

22. Daneshvar, P.; Hariri, M.; Ghiasvand, R.; Askari, G.; Darvishi, L.; Mashhadi, N.S.; Khosravi-boroujeni, H. Effect of eight weeks of quercetin supplementation on exercise performance, muscle damage and body muscle in male badminton players. Int. J. Prev. Med. 2013, 4, S53. [PubMed]

23. Edwards, R.L.; Lyon, T.; Litwin, S.E.; Rabovsky, A.; Symons, J.D.; Jalili, T. Quercetin reduces blood pressure in hypertensive subjects. J. Nutr. 2007, 137, 2405-2411. [CrossRef] [PubMed]

24. Egert, S.; Wolffram, S.; Bosy-Westphal, A.; Boesch-Saadatmandi, C.; Wagner, A.E.; Frank, J.; Rimbach, G.; Mueller, M.J. Daily quercetin supplementation dose-dependently increases plasma quercetin concentrations in healthy humans. J. Nutr. 2008, 138, 1615-1621. [CrossRef] [PubMed]

25. Ferry, D.R.; Smith, A.; Malkhandi, J.; Fyfe, D.W.; deTakats, P.G.; Anderson, D.; Baker, J.; Kerr, D.J. Phase I clinical trial of the flavonoid quercetin: Pharmacokinetics and evidence for in vivo tyrosine kinase inhibition. Clin. Cancer Res. 1996, 2, 659-668.

26. Katske, F.; Shoskes, D.A.; Sender, M.; Poliakin, R.; Gagliano, K.; Rajfer, J. Treatment of interstitial cystitis with a quercetin supplement. Tech. Urol. 2001, 7, 44-46.

27. Lee, K.-H.; Park, E.; Lee, H.-J.; Kim, M.-O.; Cha, Y.-J.; Kim, J.-M.; Lee, H.; Shin, M.-J. Effects of daily quercetin-rich supplementation on cardiometabolic risks in male smokers. Nutr. Res. Pract. 2011, 5, $28-33$. [CrossRef]

28. Lozoya, X.; Reyes-Morales, H.; Chávez-Soto, M.A.; Martínez-García, M.d.C.; Soto-González, Y.; Doubova, S.V. Intestinal anti-spasmodic effect of a phytodrug of Psidium guajava folia in the treatment of acute diarrheic disease. J. Ethnopharmacol. 2002, 83, 19-24. [CrossRef]

29. Shoskes, D.A.; Zeitlin, S.I.; Shahed, A.; Rajfer, J. Quercetin in men with category III chronic prostatitis: A preliminary prospective, double-blind, placebo-controlled trial. Urology 1999, 54, 960-963. [CrossRef]

30. Rear, R.; Bell, R.M.; Hausenloy, D.J. Contrast-induced nephropathy following angiography and cardiac interventions. Heart 2016, 102, 638-648. [CrossRef]

31. Martens, R.J.H.; Houben, A.J.H.M.; Kooman, J.P.; Berendschot, T.T.J.M.; Dagnelie, P.C.; van der Kallen, C.J.H.; Kroon, A.A.; Leunissen, K.M.L.; van der Sande, F.M.; Schaper, N.C.; et al. Microvascular endothelial dysfunction is associated with albuminuria: The Maastricht Study. J. Hypertens. 2018, 36, 1178-1187. [CrossRef] [PubMed]

32. Yuksel, Y.; Yuksel, R.; Yagmurca, M.; Haltas, H.; Erdamar, H.; Toktas, M.; Ozcan, O. Effects of quercetin on methotrexate-induced nephrotoxicity in rats. Hum. Exp. Toxicol. 2017, 36, 51-61. [CrossRef] [PubMed]

33. Levin, A.; Pate, G.E.; Shalansky, S.; Al-Shamari, A.; Webb, J.G.; Buller, C.E.; Humphries, K.H. N-acetylcysteine reduces urinary albumin excretion following contrast administration: Evidence of biological effect. Nephrol. Dial. Transplant. 2007, 22, 2520-2524. [CrossRef] [PubMed]

34. Bolisetty, S.; Agarwal, A. Urine albumin as a biomarker in acute kidney injury. Am. J. Physiol. Ren. Physiol. 2011, 300, F626-F627. [CrossRef] [PubMed]

35. Rudnick, M.R.; Goldfarb, S.; Wexler, L.; Ludbrook, P.A.; Murphy, M.J.; Halpern, E.F.; Hill, J.A.; Winniford, M.; Cohen, M.B.; VanFossen, D.B. Nephrotoxicity of ionic and nonionic contrast media in 1196 patients: A randomized trial. Kidney Int. 1995, 47, 254-261. [CrossRef] [PubMed]

36. Schwab, S.J.; Hlatky, M.A.; Pieper, K.S.; Davidson, C.J.; Morris, K.G.; Skelton, T.N.; Bashore, T.M. Contrast nephrotoxicity: A randomized controlled trial of a nonionic and an ionic radiographic contrast agent. N. Engl. J. Med. 1989, 320, 149-153. [CrossRef] [PubMed]

37. Jungbauer, C.G.; Uecer, E.; Stadler, S.; Birner, C.; Buchner, S.; Maier, L.S.; Luchner, A. N-acteyl- $\backslash$ s s-D-glucosaminidase and kidney injury molecule-1: New predictors for long-term progression of chronic kidney disease in patients with heart failure. Nephrology 2016, 21, 490-498. [CrossRef]

38. Sheira, G.; Noreldin, N.; Tamer, A.; Saad, M. Urinary biomarker N-acetyl- $\beta$-D-glucosaminidase can predict severity of renal damage in diabetic nephropathy. J. Diabetes Metab. Disord. 2015, 14, 4. [CrossRef] [PubMed]

39. Han, W.K.; Waikar, S.S.; Johnson, A.; Betensky, R.A.; Dent, C.L.; Devarajan, P.; Bonventre, J.V. Urinary biomarkers in the early diagnosis of acute kidney injury. Kidney Int. 2008, 73, 863-869. [CrossRef]

40. Sirota, J.C.; Klawitter, J.; Edelstein, C.L. Biomarkers of Acute Kidney Injury. Available online: https: //www.hindawi.com/journals/jt/2011/328120/ (accessed on 10 April 2019). 
41. Li, W.-H.; Wang, L.; He, H.-Y.; Chen, J.; Yu, Y.-R. Expression of neutrophil gelatinase-associated lipocalin in low osmolar contrast-induced nephropathy in rats and the effect of $\mathrm{N}$-acetylcysteine. Exp. Ther. Med. 2016, 12, 3175-3180. [CrossRef]

42. Bonventre, J.V. Kidney injury molecule-1 (KIM-1): A urinary biomarker and much more. Nephrol. Dial. Transpl. 2009, 24, 3265-3268. [CrossRef] [PubMed]

43. Huxley, R.R.; Neil, H.A.W. The relation between dietary flavonol intake and coronary heart disease mortality: A meta-analysis of prospective cohort studies. Eur. J. Clin. Nutr. 2003, 57, 904. [CrossRef] [PubMed]

44. Kim, G.-H. Renal Effects of Prostaglandins and Cyclooxygenase-2 Inhibitors. Electrolytes Blood Press. 2008, 6, 35-41. [CrossRef] [PubMed]

45. Hollman, P.C.; de Vries, J.H.; van Leeuwen, S.D.; Mengelers, M.J.; Katan, M.B. Absorption of dietary quercetin glycosides and quercetin in healthy ileostomy volunteers. Am. J. Clin. Nutr. 1995, 62, 1276-1282. [CrossRef]

46. Levey, A.S.; Coresh, J.; Greene, T.; Stevens, L.A.; Zhang, Y.L.; Hendriksen, S.; Kusek, J.W.; Van Lente, F. Using standardized serum creatinine values in the modification of diet in renal disease study equation for estimating glomerular filtration rate. Ann. Intern. Med. 2006, 145, 247-254. [CrossRef]

47. Bradford, M.M. A rapid and sensitive method for the quantitation of microgram quantities of protein utilizing the principle of protein-dye binding. Anal. Biochem. 1976, 72, 248-254. [CrossRef]

48. Laskey, W.K.; Jenkins, C.; Selzer, F.; Marroquin, O.C.; Wilensky, R.L.; Glaser, R.; Cohen, H.A.; Holmes, D.R.; Investigators, N.D.R. Volume-to-creatinine clearance ratio: A pharmacokinetically based risk factor for prediction of early creatinine increase after percutaneous coronary intervention. J. Am. Coll. Cardiol. 2007, 50, 584-590. [CrossRef]

49. Yamamoto, M.; Hayashida, K.; Mouillet, G.; Chevalier, B.; Meguro, K.; Watanabe, Y.; Dubois-Rande, J.-L.; Morice, M.-C.; Lefèvre, T.; Teiger, E. Renal function-based contrast dosing predicts acute kidney injury following transcatheter aortic valve implantation. JACC Cardiovasc. Interv. 2013, 6, 479-486. [CrossRef]

50. Sadat, U.; Usman, A.; Boyle, J.R.; Hayes, P.D.; Solomon, R.J. Contrast medium-induced acute kidney injury. Cardiorenal Med. 2015, 5, 219-228. [CrossRef]

(C) 2019 by the authors. Licensee MDPI, Basel, Switzerland. This article is an open access article distributed under the terms and conditions of the Creative Commons Attribution (CC BY) license (http://creativecommons.org/licenses/by/4.0/). 